\title{
REVIEW
}

\section{Acute promyelocytic leukemia: where did we start, where are we now, and the future}

\author{
CC Coombs ${ }^{1}$, M Tavakkoli $^{1}$ and MS Tallman ${ }^{1,2}$
}

\begin{abstract}
Historically, acute promyelocytic leukemia (APL) was considered to be one of the most fatal forms of acute leukemia with poor outcomes before the introduction of the vitamin A derivative all-trans retinoic acid (ATRA). With considerable advances in therapy, including the introduction of ATRA initially as a single agent and then in combination with anthracyclines, and more recently by development of arsenic trioxide (ATO)-containing regimens, APL is now characterized by complete remission rates of $90 \%$ and cure rates of $\sim 80 \%$, even higher among low-risk patients. Furthermore, with ATRA-ATO combinations, chemotherapy may safely be omitted in low-risk patients. The disease is now considered to be the most curable subtype of acute myeloid leukemia (AML) in adults. Nevertheless, APL remains associated with a significant incidence of early death related to the characteristic bleeding diathesis. Early death, rather than resistant disease so common in all other subtypes of AML, has emerged as the major cause of treatment failure.
\end{abstract}

Blood Cancer Journal (2015) 5, e304; doi:10.1038/bcj.2015.25; published online 17 April 2015

\section{INTRODUCTION}

Acute promyelocytic leukemia (APL) is a unique subtype of acute myeloid leukemia (AML), with the first description as a distinct entity in $1957 .{ }^{1}$ The disease is identified by distinctive morphology and is distinguished by a balanced reciprocal translocation between chromosomes 15 and 17. Historically, APL has been characterized by a rapidly fatal course with a high incidence of early hemorrhagic death. This became evident in early studies when patients who were untreated or received corticosteroids experienced a median survival of $<1$ week, ranging from 1 day to 1 month. ${ }^{2-6}$ Current recommendations are that when a diagnosis of APL is suspected based upon clinical presentation and/or morphology, the disease should be treated as a medical emergency. Urgent administration of ATRA should be initiated with aggressive supportive measures including blood product support with platelets and cryoprecipitate while the genetic diagnosis is rapidly established.

Risk stratification is imperative in the treatment of APL patients, as those with low-risk disease (white blood cell count (WBC) $\leq 10000 / \mu \mathrm{l})$ are generally treated with less intensive regimens than those patients presenting with high-risk disease (WBC $>10$ $000 / \mu \mathrm{l})$. Sanz et $a l^{8}{ }^{8}$ initially defined patients with WBC $\leq 10000 / \mu \mathrm{l}$ and platelet count $>40000 / \mu \mathrm{l}$ as low risk for relapse, WBC $\leq 10$ $000 / \mu \mathrm{l}$ and platelet count $\leq 40 \mathrm{k}$ as intermediate risk and WBC $>10000 / \mu \mathrm{l}$ as high risk. However, as the outcomes for patients with low- and intermediate-risk disease are similar, these categories have been collapsed into one and considered as lowrisk disease. In the past two decades, therapy for newly diagnosed APL has evolved from an all-trans retinoic acid (ATRA)+chemotherapy backbone for all patients to the addition of arsenic trioxide (ATO) to ATRA with omission of chemotherapy in low-risk patients as a new standard of care.

\section{WHERE DID WE START}

Induction regimens

APL has been associated with a high incidence of early hemorrhagic death. Early studies with induction including 6-mercatopurine (6-MP) alone or in combination with steroids, methyl-glyoxal guanyl hydrazine and/or methotrexate led to poor results. ${ }^{9}$ In the largest studies, remission rates were $5-14 \%$, with survival ranging from 3 to 16 weeks (median 3.5 weeks) among all patients, and 4 months to $>6$ years among responders. ${ }^{9-14}$ Despite waning beliefs that a cure could be attained, by the 1970s, anthracyclines were shown to yield complete remission (CR) rates that were at least comparable to, if not better than, those of other AML subtypes. ${ }^{9,15-17}$

In 1973, daunorubicin (DNR) was shown to increase remission rates from 13 to $58 \%$ and to reduce hemorrhage-related mortality after 5 days of therapy relative to 6-MP-based regimens. ${ }^{9}$ It was also shown to induce durable remissions (median 26 months). ${ }^{9}$ Numerous investigators subsequently validated the efficacy of DNR in APL. ${ }^{14-26}$ Exceptional outcomes were later reported with higher dosing regimens (61\% survival at 9 years, no relapses after 3 years). ${ }^{22}$ In addition, lower rates of death (41\% vs $76 \%$ ) and relapse ( $10 \%$ vs $68 \%$ ) were reported in patients $<50$ years of age with increasing DNR doses $\left(180-210\right.$ vs $\left.40-135 \mathrm{mg} / \mathrm{m}^{2}\right) .^{22}$

Given the efficacy of DNR as a single agent, investigators sought to identify the superiority of anthracycline drug combinations over DNR alone. DNR was reported to yield similar rates of CR $(67 \%$ vs $58 \%, P$ not significant) and early hemorrhagic death (10\% vs $9 \%, P$ not significant) compared with various DNR and doxorubicin drug combinations in an analysis of 268 patients, although this analysis is limited by small numbers and its retrospective nature. ${ }^{20}$ The Southwest Oncology Group showed similar patient outcomes even with the addition of other chemotherapeutic agents to DNR. $^{22}$ Despite similar rates of $\mathrm{CR}(73 \%)$ and death during

${ }^{1}$ Memorial Sloan Kettering Cancer Center, Leukemia Service, New York, NY, USA and ${ }^{2}$ Weill Cornell Medical College, New York, NY, USA. Correspondence: Dr CC Coombs, Memorial Sloan Kettering Cancer Center, Weill Cornell Medical College, 1275 York Avenue, New York, NY 10065, USA.

E-mail: coombsc@mskcc.org

Received 5 February 2015; accepted 13 February 2015 
induction (27\%), Petti et al. ${ }^{18}$ reported more rapid responses (23 vs 45 days), longer durations of remission (14 vs 7 months) and better survival rates $(27 \%,>6$ years vs $0 \%>25$ months) with DNR as a single agent relative to DNR-based drug combinations, limiting enthusiasm for combination therapy in these early studies.

Pre-ATRA era reinduction, consolidation and maintenance therapy Several early studies attempted to optimize reinduction for relapsed patients, consolidation and maintenance strategies in APL. Initial reports addressing outcomes following relapse were poor, with the best outcomes being achieved by Kantarjian et al. ${ }^{21}$ who reported a second CR rate (CR2) of $53 \%$ utilizing various reinduction regimens including combinations of doxorubicin, cytarabine, vincristine, amsacrine and prednisone. Cunningham et $a l .{ }^{15}$ reported a median survival of 6 weeks following relapse. A variety of reinduction attempts were utilized in the pre-ATRA era; strategies including previous induction regimens were rarely successful. ${ }^{15,27}$ Allogeneic hematopoietic stem cell transplantation (HSCT) yielded poor results during first CR (CR1); however, allogeneic and autologous HSCT resulted in the longest CR2 durations (29 to $48+$ months). ${ }^{15,21,27}$ Furthermore, unlike other subtypes of $A M L$, it had been recognized in the pre-ATRA era that specific maintenance regimens were shown to be critical to longterm survival. Of the patients, $42 \%$ receiving POMP (6-MP, methotrexate, vincristine and prednisone) maintenance were long-term survivors compared with $3 \%$ of those receiving cycling monthly chemotherapy. ${ }^{28}$ Kantarjian et al. ${ }^{21}$ also observed reduced remission durations when POMP maintenance was not used, $^{15,21}$ leading to support for maintenance regimens in future studies.

\section{WHERE WE ARE NOW}

Induction regimens utilizing chemotherapy: remain the standard of care in high-risk APL

ATRA was introduced clinically in 1985, and this opened a new era in the treatment of APL. ${ }^{29}$ ATRA induces differentiation of leukemic promyelocytes into mature granulocytes, leading to its evaluation either as a single agent or in combination with chemotherapy, first in relapsed/refractory disease and then in newly diagnosed patients. ${ }^{30-33}$ As a single agent, ATRA induced CR rates of $85 \%$ in studies by the Shanghai group in $1988 .{ }^{33}$ The first North American Intergroup study (I0129) demonstrated a 72\% CR rate with single-agent ATRA, equivalent to rates obtained with conventional doses of cytarabine and DNR. ${ }^{30}$ However, frequent relapses were noted in patients who received ATRA alone. Continuous treatment with ATRA is characterized by reduction of its plasma concentration because of accelerated clearance. ${ }^{29}$ These findings prompted subsequent trials to combine ATRA with chemotherapy, leading to lower relapse rates.

Numerous prospective randomized studies were conducted to exploit the potential benefits of the combination of ATRA and chemotherapy. The European APL group demonstrated in a randomized study that concurrent ATRA plus chemotherapy (DNR and cytarabine) resulted in a lower relapse rate at 2 years (6\% vs $16 \%, P=0.04)^{34}$ when compared with sequential ATRA followed by chemotherapy, and this has been confirmed in other large multicenter trials. ${ }^{35-39}$ Furthermore, the early addition of chemotherapy to ATRA decreased the incidence of retinoic acid syndrome. ${ }^{40}$ Ultimately, these studies established concurrent ATRA and anthracycline-based chemotherapy (either an anthracycline plus cytarabine or an anthracycline alone) as the standard of care for induction in newly diagnosed APL patients.

There has been controversy surrounding the optimal chemotherapy regimen to combine with ATRA. First, there are no definitive data to suggest the superiority of one anthracycline over another, as no prospective studies have been conducted comparing idarubicin with DNR in APL. Furthermore, there is no clear consensus on the role of cytarabine during induction therapy, although a number of studies have indicated that cytarabine is not needed in induction in any risk subset of patients. Two randomized trials investigated the role of cytarabine combined with either idarubicin or DNR, but yielded conflicting results. ${ }^{41,42}$ The National Cancer Research Institute (NCRI) in the United Kingdom randomized patients between ATRA plus idarubicin (AIDA) and ATRA plus DNR and cytarabine (MRC AML15 trial), and reported no differences in response, relapse or overall survival (OS) rates, but less myelosuppression in the AIDA group. ${ }^{42}$ However, the study by the EuroAPL group (APL 2000) that randomized low-risk patients (age $<60$ and WBC $\leq 10000 / \mu \mathrm{l}$ ) to induction with ATRA/DNR/AraC versus ATRA/DNR and consolidation with DNR/AraC versus DNR reported an increase in 5-year cumulative incidence of relapse $(\mathrm{CIR})(13.4 \%$ vs $29 \%, P=0.01)$ and a decrease in OS rates $(92.9 \%$ vs $83.3 \%, P=0.07)$ in the group who did not receive cytarabine for induction and consolidation therapy. ${ }^{41,43}$ Subsequent prospective, nonrandomized studies by the Gruppo Italiano Malattie Ematologiche dell'Adulto (GIMEMA) and Programa Español de Tratamientos en Hematología (PETHEMA) demonstrated that AIDA is as effective in inducing remission as cytarabine-containing regimens, with $C R$ rates between 89 and 95\%. ${ }^{38,44}$ Differences in outcomes may be related to variation of individual studies, such as the consolidation regimens (ATRA vs no ATRA), the number of consolidation courses and the specific anthracycline used.

Given the favorable results from risk-adapted treatment strategies, first in the LPA99 trial followed by the LPA2005 trial, an additional induction option includes ATRA plus idarubicin with risk-adapted consolidation. ${ }^{45,46}$ Finally, with the favorable results of the APML4 trial (discussed further below), which does not include cytarabine in induction (or consolidation), an alternate approach now recommended by the National Comprehensive Cancer Network (NCCN) includes ATRA plus idarubicin and ATO. ${ }^{47}$

The introduction of ATO into the treatment of patients with APL ATO was first utilized in APL patients in the early 1990s, and led to a high $C R$ rate with relatively long-term remissions when used as a single agent. ${ }^{48}$ In preclinical models, the combination of ATRA and ATO demonstrated synergism in inducing differentiation and apoptosis, $^{49-51}$ allowing for targeted therapy of APL without exposure to chemotherapy. This synergism between ATRA and ATO has been demonstrated to eradicate APL-initiating cells through promyelocytic leukemia/retinoic acid receptor-a degradation. ${ }^{52}$ Investigators at the Shanghai Institute of Hematology performed a randomized clinical trial in which patients received ATRA, ATO or the combination of ATRA plus ATO as induction therapy. Similar CR rates between groups (between 90 and $95.2 \%$ ) were observed, but among the patients receiving combination ATRA-ATO therapy, there was a statistically significant improvement in the time to achieve $C R$, time for platelet recovery and decrease in the rate of relapse. ${ }^{53^{3}}$ The Australasian Leukaemia and Lymphoma Group (ALLG) performed a phase 2, single-armed study (APML4), reporting the outcome of 124 patients with newly diagnosed APL (23 patients with high-risk disease) treated with triple induction with ATRA, ATO and idarubicin, followed by two courses of consolidation with ATRA and ATO and 2 years of maintenance with ATRA, methotrexate and 6-MP (Figure 1). ${ }^{47}$ Outcomes were compared with historical controls from the APML3 study that used AIDA in induction and consolidation without ATO. With a median follow-up of 2 years, the 3-year OS and event-free survival (EFS) rates were $93.2 \%$ and $88.1 \%$, respectively. Compared with APML3 results, this trial demonstrated a statistically significant improvement in freedom from relapse, disease-free survival (DFS) and failure-free survival, 
a

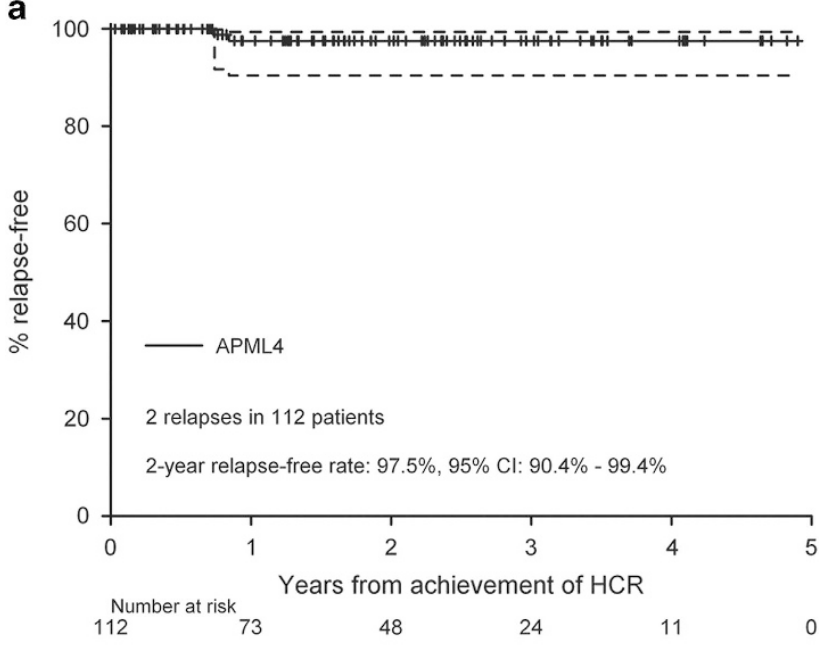

b

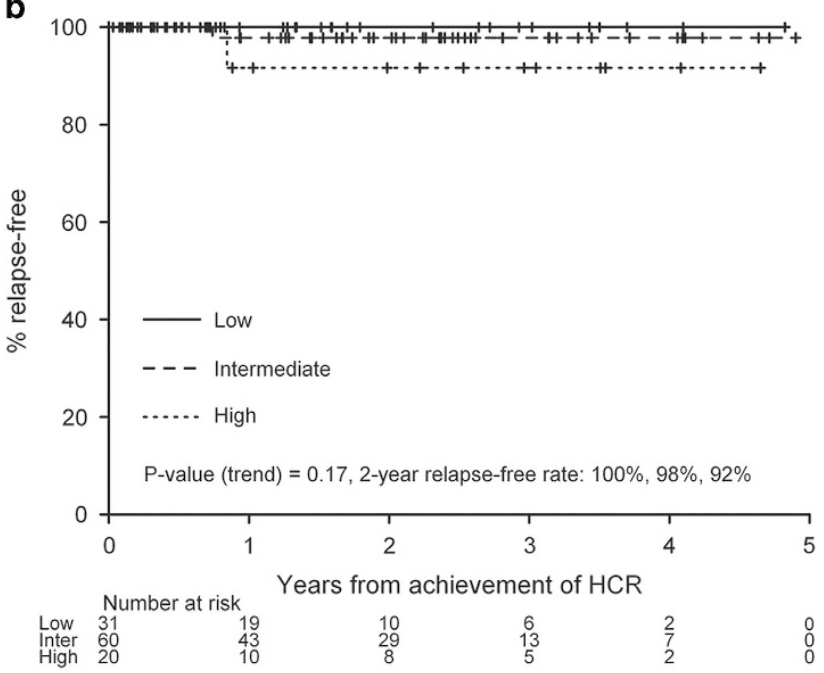

Figure 1. Relapse-free survival curves for APML4, the phase 2 trial utilizing combination of ATRA, ATO, and idarubicin in newly diagnosed APL. Panel a comprises all patients on APML4 $(n=112)$ and panel $\mathbf{b}$ stratifies patients by Sanz risk category.

but not OS (Figure 1). ${ }^{47}$ Updated results with median follow-up of 4.2 years were reported at the 2014 meeting of the American Society of Hematology, with 5-year OS and EFS rates of $94 \%$ and $90 \%$, respectively, in all risk groups $(87 \%$ and $83 \%$ in high-risk patients, respectively). ${ }^{54}$ This regimen appears very promising; although, given its phase 2 nature and comparison with historical controls, it may be premature to suggest superiority. Furthermore, given the small number of high-risk patients, dedicated randomized trials in high-risk patients are required before drawing firm conclusions regarding the optimal induction regimen in this subset of patients.

Investigators at the MD Anderson Cancer Center demonstrated that the combination treatment of ATRA and ATO is an effective treatment in untreated APL with a high CR rate of $96 \% .{ }^{55}$ However, high-risk patients (WBC $>10000 / \mu \mathrm{l}$ at presentation) achieved an inferior CR rate of $79-81 \%$ because of early treatment failure from fatal hemorrhage and differentiation syndrome despite the addition of either gemtuzumab ozogamicin (GO) or idarubicin during induction to control elevated WBC counts. ${ }^{55,56}$ This suggests this regimen may be inadequate for high-risk patients.

In summary, these studies suggested that the combination of ATRA and ATO particularly in patients with low-risk disease is very promising. However, in patients presenting with high WBC, simultaneous use of cytotoxic agents such as anthracyclines in induction appears to be important to prevent rapid development of leukocytosis, differentiation syndrome and relapse, with a possible benefit of cytarabine in consolidation, discussed further below.

The transition to nonchemotherapy-based approaches for low-risk disease: ATRA and ATO combination therapy

With the early success of ATRA- and ATO-based induction regimens, the question emerged as to whether chemotherapy could safely be eliminated or minimized to reduce treatmentassociated toxicities and long-term complications observed with cytotoxic agents. ${ }^{57}$ This effort may be particularly important as therapy-related myeloid neoplasms have been observed in APL patients. ${ }^{58-60}$ In a recent series of 918 APL patients in CR, the incidence of therapy-related myeloid neoplasms was $2.2 \%$, with the highest incidence of $5.2 \%$ in low-risk patients. ${ }^{61}$ The median OS from time of therapy-related myeloid neoplasm diagnosis in this series was 10 months; therefore, the omission of potentially leukemogenic cytotoxic chemotherapy is an attractive option to attempt to reduce the incidence of this serious complication.

Given the success of single-center studies examining the combination of ATRA with ATO as described above, a phase 3, multicenter trial comparing ATRA plus idarubicin with ATRA plus ATO was conducted in patients with low- to intermediate-risk APL. In July 2013, Lo-Coco and colleagues ${ }^{62}$ published results of this trial, with average follow-up of 33.4 months with extended results of the final series of 276 patients presented at the 2014 American Society of Hematology meeting. The study was designed as a noninferiority trial to demonstrate that the rate of EFS between the groups was not $>5 \%$. The 2--year EFS rates were $97 \%$ in the ATRA-ATO group, and $86 \%$ in the ATRA-chemotherapy group meeting a $P<0.001$ for noninferiority and a $P=0.02$ for superiority, with EFS $98 \%$ vs $85 \%$ on updated series $(P=0.0002)$ (Figure 2). ${ }^{62}$ The 2 -year OS probability was $99 \%$ in the ATRA-ATO group, as compared with $91 \%$ in the ATRA-chemotherapy group $(P=0.02)$. The 2 -year DFS was $97 \%$ in the ATRA-ATO group and $90 \%$ in the ATRA-chemotherapy group $(P=0.11)$, and the 2-year CIR was $1 \%$ in the ATRA-ATO group and $6 \%$ in the ATRAchemotherapy group (CIR remained $1 \%$ in ATRA-ATO but increased to $9.4 \%$ for ATRA-chemotherapy in the updated analysis) ( $P=0.24$ on initial analysis ${ }^{63}$ and $P=0.005$ in the updated analysis) (Figure 2). ${ }^{62}$ Toxicities differed between the two arms, in that hematologic toxicity occurred more frequently in the ATRAchemotherapy arm, but hepatic toxicity and prolongation of the QTC interval occurred more frequently in the ATRA-ATO arm. Importantly, there was no difference in the incidence of differentiation syndrome between the arms, possibly related to the use of prophylactic prednisone in both groups. ${ }^{63}$ Healthrelated quality of life for fatigue severity was statistically improved in the ATRA-ATO arm as compared with ATRA-chemotherapy. ${ }^{64}$

In summary, ATRA-ATO was noninferior and possibly superior to ATRA-chemotherapy. The observed improvement in EFS and OS in the ATRA-ATO arm without significant differences in DFS and CIR suggests that these regimens have similar antileukemic efficacy, but with lower mortality in the ATRA-ATO arm from causes other than relapse. ${ }^{63}$ Longer-term follow-up will be important to draw final conclusions regarding efficacy and longterm toxicity.

Eghtedar et al. ${ }^{65}$ recently examined the incidence of secondary malignancies in patients treated with ATRA-ATO $(n=106$, with median follow-up of 29 months) versus ATRA-idarubicin ( $n=54$, with median follow-up of 136 months). Nine patients in the chemotherapy group developed secondary malignancies compared with two patients in the ATRA-ATO group. They concluded that the treatment of APL patients using ATRA-ATO is not 
4
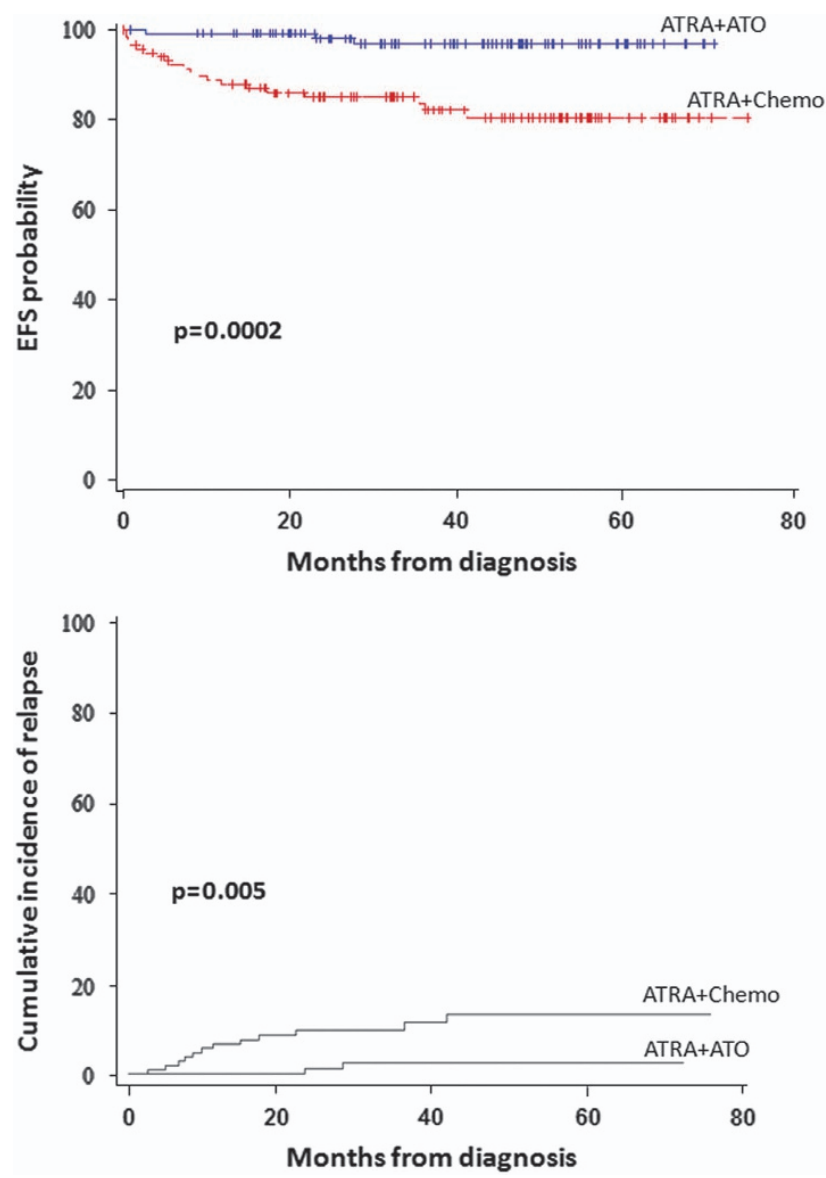

Figure 2. EFS probability and $\mathrm{CIR}$ in non-high-risk $A P L$ patients on Italian-German APL 0406 trial comparing ATRA-ATO with ATRAchemotherapy on the extended final series. ${ }^{62}$

associated with a higher incidence of secondary malignancies with a $P=0.29$, adjusted for unit of time exposure. Longer followup of randomized populations such as the phase 3 study by Lo-Coco et al. ${ }^{63}$ would provide more useful estimations regarding long-term toxicities such as secondary malignancies.

Based upon these favorable results of the phase 3 trial comparing ATRA-ATO with ATRA-chemotherapy, ATRA-ATO has emerged as the new standard of care for patients with low-(tointermediate) risk APL. Furthermore, ATRA-ATO therapy also may serve as an attractive alternative for patients who are considered unfit for conventional treatment and with severe comorbidities, such as older adults and patients with cardiac dysfunction or other severe organ dysfunction.

Consolidation therapy: risk-adapted approach

Historical comparisons of trials by the GIMEMA ${ }^{66}$ and PETHEMA ${ }^{45}$ have demonstrated a lower relapse rate $(8.7 \%$ vs $20.1 \%)$ and higher DFS and OS rates with concomitant administration of ATRA with chemotherapy in consolidation. However, no randomized studies have demonstrated this benefit of ATRA. Nevertheless, this approach has been routinely adopted.

There is no consensus regarding which specific chemotherapy is optimal in consolidation. The focus of past research efforts has been to develop risk-adapted strategies to provide more intensive treatment in high-risk patients with WBC $>10000 / \mu$ l while minimizing toxicities in low-risk patients. A cooperative group multicenter study by PETHEMA (LPA2005) administered cytarabine only in high-risk patients, achieving a lower CIR at 3 years ( $11 \%$ vs
$26 \%, P=0.03)$ compared with historical controls from LPA99 trial. ${ }^{46}$ Similarly, GIMEMA (AIDA2000) administered cytarabine in high-risk patients only and reported an improved incidence of relapse at 6 years in this group $(9.3 \%$ vs $49.7 \%, P<0.001)$ compared with historical controls (AIDA0493). ${ }^{66}$ However, the improved outcome observed in the GIMEMA study is likely related to the use of ATRA in consolidation as the historical comparator received chemotherapy without ATRA. In contrast, a study by the NCRI, published only in abstract form, demonstrated no benefit of cytarabine in all risk groups of patients. ${ }^{42}$ Taken together, the majority of studies suggest a benefit of cytarabine in high-risk patients, possibly because of the synergistic effect of the combination of ATRA plus cytarabine. $^{67}$ However, taking contemporary studies utilizing ATRA-ATO combination into account, it appears that cytarabine can be omitted in low-risk patients in consolidation and excellent outcome is preserved. ${ }^{63}$

To reduce chemotherapy exposure in low-risk patients, multiple cooperative groups have investigated the role of ATRA and ATO in consolidation. The North American Intergroup trial (C9710) randomized patients to receive two cycles of consolidation with ATRA plus DNR, either immediately following induction therapy or preceded by two 25-day cycles of ATO. ${ }^{68}$ The results demonstrated that for all risk groups, ATO in consolidation significantly improved 3-year DFS (90\% vs 70\%, $P=<0.0001)$; and there was a nonstatistically significant improvement in OS $(86 \%$ vs $81 \%$, $P=0.07) .{ }^{68}$ In a phase 2 study, Gore et al. ${ }^{69}$ reported comparable outcomes (DFS 90 and OS 88\%) with considerably reduced amount of anthracyclines combined with a single cycle of ATO. Other groups have completely eliminated cytotoxic chemotherapy and investigated the role of ATO either as a single agent or combined with ATRA in consolidation. Using ATRA-ATO, with GO as alternate therapy for patients with toxicity to ATRA-ATO, investigators at the MD Anderson Cancer Center reported a 3-year OS of $85 \% .^{70}$ The ALLG reported a 3 -year OS and EFS rates of $93 \%$ and $87 \%$, respectively, utilizing ATRA-ATO in consolidation in APML4. ${ }^{47}$ Finally, the phase 3 trial by Lo Coco et al. ${ }^{63}$ demonstrated the utility of ATRA-ATO in consolidation for standard-risk patients, yielding at a minimum noninferior, and possibly superior, outcomes, as outlined above.

\section{Maintenance therapy}

Prolonged maintenance therapy is typically included in modern APL treatment protocols, although its importance remains controversial. A Cochrane review examining published, ongoing and unpublished clinical trials through July 2012 sought to determine the role for maintenance therapy in APL in CR1. Selection criteria required randomized controlled trials assessing maintenance treatment in patients with newly diagnosed APL in CR1 following induction or induction and consolidation. Ten randomized trials enrolling 2072 patients were included in the systematic review, and meta-analysis was conducted on nine of these trials. There was no statistically significant improvement in OS in the comparisons examined (maintenance treatment vs observation, ATRA maintenance vs non-ATRA maintenance, ATRA maintenance alone vs ATRA with chemotherapy maintenance). ${ }^{71}$ However, DFS was improved with any maintenance compared with observation (hazard ratio $0.59,95 \%$ confidence interval 0.48 0.74 with 1209 patients in 5 trials), although DFS was not statistically improved with ATRA-based regimens compared with non-ATRA regimens (hazard ratio $0.72,95 \%$ confidence interval 0.51-1.01 with 670 patients from 4 trials). ${ }^{71}$ Although suggestive that maintenance may improve DFS, though not OS, in APL, the significant heterogeneity with regard to specific induction and consolidation regimens between these trials limits the generalized applicability of these findings.

Coutre et al. ${ }^{72}$ recently reported the results of the trial S0521 that randomized low-risk patients who achieved a molecular CR to 
maintenance with ATRA, 6-MP and methotrexate vs observation; all patients received standard induction of DNR, ATRA and cytarabine and consolidation with two courses each of ATO and DNR/cytarabine. Enrollment was stopped because of slow accrual. However, of the 68 patients randomized, no relapses were observed at median follow-up of 36.1 months, suggesting that in patients receiving intensive induction/consolidation including ATO, maintenance may not be necessary.

Relapsed-refractory APL

With modern therapy, relapsed/refractory APL is a rare condition, as $90 \%$ of patients achieve CR after initial therapy and $80 \%$ of patients are cured of their disease. Delayed CR (that is, CR after 35 days of therapy) has been associated with a higher rate of relapse $(31 \%$ vs $17 \%, P=0.001) .^{73}$ Failure to achieve remission after ATRA-based induction therapy is rare, largely restricted to rare patients with ATRA-resistant variants, such as PLZF-RARApositive APL. ${ }^{74}$ Resistance to ATO has recently been described in a series of 13 ATO-resistant APL patients using direct sequencing, 9 of whom harbored $P M L$ mutations, and 7 of these simultaneously harbored RARA mutations. ${ }^{75}$

Relapse occurs in $5-20 \%$ of patients, with $<3 \%$ of patients with low-risk disease relapsing, but closer to $20 \%$ relapse rate in some series among high-risk patients, although this rate appears to be lower at $\sim 10-12 \%$ in contemporary series. ${ }^{47,76}$ Relapse at extramedullary sites is an increasingly recognized problem, occurring in $3-5 \%$ of patients. ${ }^{77}$ Therapeutic options for relapsed/refractory APL have included ATO, thought to be the single most active agent in APL, with 40 of 47 relapsed APL patients achieving $C R$ in an early study. ${ }^{48}$ Further treatment options for induction include combinations of ATO with chemotherapy such as anthracyclines and anti-CD33 humanized antibodies (discussed further below).

However, as ATO moves to front-line therapeutic strategies, the response to ATO in relapse to those patients previously exposed to ATO is unclear. This issue will become an important one, although for increasingly fewer patients. A retrospective study examined 64 consecutive first-relapsed APL patients receiving salvage therapy with ATO and chemotherapy, 52 of whom had a hematologic relapse. Of patients with hematologic relapse, 20 had relapsed after previous ATO therapy and 32 did not receive prior ATO therapy. ${ }^{78}$ There was no statistical difference between CR2 rate $(80 \%$ vs $93.8 \%, P=0.189)$ or 4 -year OS rate $(62.4 \%$ vs $71.2 \%$, $P=0.816)$, but there was a statistically significant difference between relapse rate $(68.8 \%$ vs $33.3 \%, P=0.03)$ and 4-year relapse-free survival rate $(29.8 \%$ vs $66.2 \%, P=0.023) .{ }^{78}$ This study is limited by its retrospective design and small number of patients. Larger prospective studies may help elucidate the utility of rechallenge with ATO in previously exposed patients.

Once a patient has achieved CR2, HSCT is considered in patients who are candidates. Autologous as well as allogeneic transplants have been evaluated. ${ }^{79}$ Although both have been associated with durable remission and prolonged survival, the former approach has led to the best outcomes in all comparative studies. A phase 2 study of 35 patients evaluating the efficacy and feasibility of induction and consolidation with ATO followed by auto-HSCT in relapsed APL demonstrated a 5 -year EFS of $65 \%$ and a 5 -year OS of $77 \% .^{80}$ Recent data suggest an improved 5 -year DFS and OS in auto-HSCT when compared with allo-HSCT (DFS 63\% in auto-HSCT and $50 \%$ in allo-HSCT $(P=0.10)$; OS $75 \%$ in auto-HSCT vs $54 \%$ in allogeneic $(P=0.002)){ }^{81}$ In a retrospective study that reviewed patients who received ATO-based therapy before auto-HSCT, a delay in neutrophil recovery has been demonstrated, although the clinical significance is uncertain. ${ }^{82}$ Owing to the increasing use of ATO in front-line therapy for APL, larger prospective studies are necessary to validate such findings and to understand the mechanism of delayed neutrophil recovery. ${ }^{82}$

\section{THE FUTURE}

Efforts to improve early death rate

Unlike other subtypes of AML, the primary cause of treatment failure in patients with APL is early death, defined as death within the first 30 days of diagnosis. Although the rate of early death is low in patients enrolled on clinical trials, it is significantly higher in patients who are not enrolled on trials, likely related to selection bias $\left(20-30 \%\right.$ compared with $3 \%$ in a recent study $\left.{ }^{83}\right)$. Early death is particularly common in older patients. ${ }^{84}$ The observed improvement in early death rate over time is modest at best, decreasing from $22.1 \%$ in $1992-1995$, to $14.7 \%$ in $1996-2001$ and $17.5 \%$ between 2002 and 2007, in a population-based study. ${ }^{85}$ The reasons for early deaths in APL are multiple, although death during induction is most frequently related to the hemorrhagic diathesis because of hyperfibrinolysis, proteolysis and disseminated intravascular coagulation, further complicated by thrombocytopenia. ${ }^{86}$ Delays in ATRA therapy have been suggested as a contributing factor in early deaths, with ATRA ordered in only $31 \%$ of APL on the day the diagnosis was suspected in one retrospective analysis. ${ }^{87}$ In another retrospective review examining early APL deaths, delay in ATRA administration was not a statistically significant cause for early death, although interpretation of these data is limited as the group with delayed ATRA therapy was generally less sick than the group that received ATRA promptly. ${ }^{88}$

Ultimately, given excellent response rates in APL with low relapse rates even among high-risk patients, improvement in the early death rate in APL is of paramount importance. Education of medical providers should lead to a high level of vigilance regarding this diagnosis, to facilitate prompt suspicion for the diagnosis of APL, at which time ATRA should be initiated in addition to aggressive supportive measures. There is general consensus regarding aggressive blood product support, in that platelets should be maintained above $30-50 \times 10^{9} / \mathrm{l}$ and fibrinogen above $100-150 \mathrm{mg} / \mathrm{dl}^{89}$

\section{Novel agents}

Oral ATO. In the aforementioned studies examining therapy with ATO, the intravenous (i.v.) formulation was utilized. The use of i.v. ATO is inconvenient, as it requires frequent patient visits for administration and maintenance of vascular access, further complicated by an observed increase in the rate of central venous catheter-associated thrombosis among APL patients compared with acute lymphocytic leukemia and AML patients. ${ }^{90} \mathrm{An}$ oral formulation of ATO has been developed that showed favorable oral absorption with an achieved bioavailability of up to $95 \%$ of an equivalent dose of i.v. ATO.91 Oral ATO was first utilized in the treatment of relapsed APL that showed high efficacy and similar toxicity profile to i.v. formulations. ${ }^{92}$ Notably, the QTc prolongation and ventricular arrhythmias seen with i.v. ATO were not observed with oral ATO, likely because of lower peak plasma arsenic concentrations achieved with oral formulations. ${ }^{93}$ Oral ATO has since been tested in the setting of maintenance after first CR, and with 10-year follow-up, this regimen appears to have similar outcomes to i.v. formulations. ${ }^{94}$ Finally, oral ATO versus i.v. ATO in combination with ATRA was examined in a randomized, phase 3 noninferiority trial, and oral ATO with ATRA was noninferior to i.v. ATO with ATRA (Figure 3). ${ }^{95}$ In summary, the oral formulation of ATO exhibit excellent activity and combinations with ATRA provide an opportunity for a completely oral, chemotherapy-free regimen for treating APL. ${ }^{96}$ Although oral ATO is an attractive therapeutic approach, longer-term follow-up is needed, and it is not yet readily available in the United States.

Anti-CD33 monoclonal antibodies. GO is an anti-CD33 monoclonal antibody conjugated to the toxin calicheamicin, and has shown significant activity in APL because of the high level of 


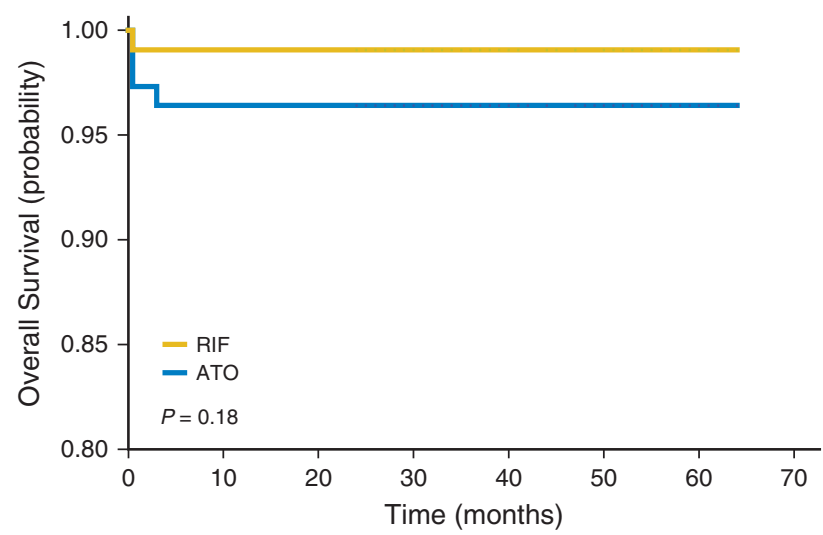

Figure 3. Overall survival curves for phase 3 randomized noninferiority trial comparing oral ATO (realgar-indigo naturalis formula (RIF) with i.v. ATO. ${ }^{95}$

expression of CD33 target antigen on APL cells. ${ }^{97}$ However, safety concerns led to the US marketing withdrawal of GO in June 2010, although this decision has more recently been called into question. ${ }^{98,99} \mathrm{GO}$ is currently available under compassionate use programs. SGN-CD33A is a next-generation anti-CD33 antibody currently in clinical trials for $A M L$, including $A P L$, that has demonstrated antileukemic activity with $47 \%$ blast clearance in interim analysis of the phase 1 study. ${ }^{100}$

Lo-Coco et al. ${ }^{101}$ explored the use of GO as a single agent in relapsed APL. Of the 16 patients treated, a molecular remission was obtained in 11 patients after two doses, and in an additional two patients after the third dose. One patient achieved molecular remission after first dose but was taken off drug because of hepatic toxicity. The last two patients had disease progression during treatment. These results supported that GO has significant single-agent activity in relapsed APL. ${ }^{101}$

Ravandi et al. $^{70}$ published the MD Anderson experience of utilizing ATRA-ATO induction with the addition of GO in high-risk, newly diagnosed APL patients (WBC $\geq 10000 / \mu$ lat presentation in all patients, or WBC $>30000 / \mu$ l during induction in the second cohort of patients). Post-remission therapy consisted of ATRA and ATO, with GO given if either ATRA or ATO were discontinued because of toxicity. In the 82 patients examined, 74 achieved a CR with one additional $C R$ with incomplete platelet recovery. The CR rate for low-risk patients was $95 \%$ and CR rate in high-risk patients was $81 \%^{70}$

Tamibarotene. Tamibarotene (formerly called Am80) is a synthetic retinoid that induces differentiation of HL-60 and NB-4 cells with $\sim 10$ times more potent in vitro activity compared with ATRA, with a favorable pharmacokinetic profile as the plasma level does not decline after daily administration. ${ }^{102,103}$ A phase 3 study was conducted to compare tamibarotene with ATRA as maintenance therapy for patients with newly diagnosed APL. Of the 344 eligible patients, 319 (93\%) achieved CR with 269 undergoing maintenance randomization after completing three courses of consolidation. ${ }^{104}$ There was no statistical difference between ATRA and tamibarotene for relapse-free survival, although in an exploratory analysis, high-risk patients were noted to have an improved relapse-free survival rate of $87 \%$ in the tamibarotene arm as compared with $58 \%$ in the ATRA arm. ${ }^{104}$ Tamibarotene was examined as a single agent for induction in relapsed/refractory $A P L$, showing activity in patients who previously received ATRA and ATO; however, responses were not durable. ${ }^{105}$ Ultimately, the utility of this agent in the ATO era is of uncertain significance, although it possibly may have a role in high-risk patients for maintenance therapy, but this would needs to be confirmed in larger, dedicated studies.

\section{Survivorship}

Given the exceedingly high cure rate with modern therapy and the relatively young median age of patients, a future focus should emphasize optimization of survivorship care for APL patients. In a recent series, outcomes for APL patients treated with ATRA-ATO and ATRA-chemotherapy who were in CR for at least 3 years were retrospectively examined, revealing an $8 \%$ incidence of second malignancies in addition to the development of comorbid conditions such as diabetes mellitus, hypertension and cardiac disease, emphasizing the importance of long-term follow-up for APL survivors. ${ }^{106}$

\section{CONCLUSION}

APL has been transformed from the most fatal to the most curable form of acute leukemia in adults. The standard of care for low-risk patients no longer includes chemotherapy given the success of the phase 3 noninferiority trial examining ATRA-ATO combination therapy. Regimens for treating high-risk APL have not been sufficiently compared to suggest superiority of one regimen over another. Given the tolerability and excellent long-term outcomes, our approach for high-risk patients includes triple induction with ATRA, ATO and idarubicin. Areas of ongoing need include efforts to decrease the early death rate, which is the primary cause for treatment failure, refinements in strategies for high-risk patients and a focus on survivorship care.

APL has served as a paradigm for targeted, differentiationbased therapies, with ATRA and ATO changing the landscape of therapy for this once uniformly fatal disease.

\section{CONFLICT OF INTEREST}

The authors declare no conflict of interest.

\section{ACKNOWLEDGEMENTS}

Montreh Tavakkoli is a Howard Hughes Medical Institute Research Fellow.

\section{REFERENCES}

1 Hillestad LK. Acute promyelocytic leukemia. Acta Med Scand 1957; 159: 189-194.

2 Pisciotta AV, Schulz EJ. Fibrinolytic purpura in acute leukemia. Am J Med 1955; 19: 824-828.

3 Cooperberg AA, Neiman GM. Fibrinogenopenia and fibrinolysis in acute myelogenous leukemia. Ann Intern Med 1955; 42: 706-711.

4 Fisher S, Ramot B, Kreisler B. Fibrinolysis in Acute Leukemia. Israel Med J 1960; XIX: 195-198.

5 Ghitis J. Acute promyelocytic leukemia? Blood 1963; 21: 237-240.

6 Larson RA, Kondo K, Vardiman JW, Butler AE, Golomb HM, Rowley JD. Evidence for a $15 ; 17$ translocation in every patient with acute promyelocytic leukemia. $A m$ J Med 1984; 76: 827-841.

7 Sanz MA, Grimwade D, Tallman MS, Lowenberg B, Fenaux P, Estey EH et al. Management of acute promyelocytic leukemia: recommendations from an expert panel on behalf of the European LeukemiaNet. Blood 2009; 113: 1875-1891.

8 Sanz MA, Lo Coco F, Martin G, Avvisati G, Rayon C, Barbui T et al. Definition of relapse risk and role of nonanthracycline drugs for consolidation in patients with acute promyelocytic leukemia: a joint study of the PETHEMA and GIMEMA cooperative groups. Blood 2000; 96: 1247-1253.

9 Bernard J, Weil M, Boiron M, Jacquillat C, Flandrin G, Gemon MF. Acute promyelocytic leukemia: results of treatment by daunorubicin. Blood 1973; 41: 489-496.

10 Bernard PJ, Mathe G, Boulay J, Ceoard B, Chome J. La leucose aigue a promyelocytes. Schweiz Med Wochenschr 1959; 23: 604-608.

11 Baker WG, Bang NU, Nachman RL, Raafat F, Horowitz HI. Hypofibrinogenemic hemorrhage in acute myelogenous leukemia treated with heparin. With autopsy findings of widespread intravascular clotting. Ann Intern Med 1964; 61: 116-123. 
12 Rosenthal RL. Acute promyelocytic leukemia associated with hypofibrinogenemia. Blood 1963; 21: 495-508.

13 Didisheim P, Trombold JS, Vandervoort LE, Mibashan RS. Acute promyelocytic leukemia with fibrinogen and factor V deficiencies. Blood 1964; 23: 717-728.

14 Drapkin RL, Gee TS, Dowling MD, Arlin Z, McKenzie S, Kempin S et al. Prophylactic heparin therapy in acute promyelocytic leukemia. Cancer 1978; 41: 2484-2490.

15 Cunningham I, Gee TS, Reich LM, Kempin SJ, Naval AN, Clarkson BD. Acute promyelocytic leukemia: treatment results during a decade at Memorial Hospital. Blood 1989; 73: 1116-1122.

16 Sanz MA, Jarque I, Martin G, Lorenzo I, Martinez J, Rafecas J et al. Acute promyelocytic leukemia. Therapy results and prognostic factors. Cancer 1988; 61: 7-13.

17 Cordonnier C, Vernant JP, Brun B, Heilmann MG, Kuentz M, Bierling P et al. Acute promyelocytic leukemia in 57 previously untreated patients. Cancer 1985; 55: 18-25.

18 Petti MC, Avvisati G, Amadori S, Baccarani M, Guarini AR, Papa G et al. Acute promyelocytic leukemia: clinical aspects and results of treatment in 62 patients. Haematologica 1987; 72: 151-155.

19 Arlin Z, Kempin S, Mertelsmann R, Gee T, Higgins C, Jhanwar S et al. Primary therapy of acute promyelocytic leukemia: results of amsacrine- and daunorubicin-based therapy. Blood 1984; 63: 211-212.

20 Rodeghiero F, Avvisati G, Castaman G, Barbui T, Mandelli F. Early deaths and anti-hemorrhagic treatments in acute promyelocytic leukemia. A GIMEMA retrospective study in 268 consecutive patients. Blood 1990; 75: 2112-2117.

21 Kantarjian HM, Keating MJ, Walters RS, Estey EH, McCredie KB, Smith TL et al. Acute promyelocytic leukemia. Am J Med 1986; 80: 789-797.

22 Head D, Kopecky KJ, Weick J, Files JC, Ryan D, Foucar K et al. Effect of aggressive daunomycin therapy on survival in acute promyelocytic leukemia. Blood 1995; 86: $1717-1728$

23 Goldberg MA, Ginsburg D, Mayer RJ, Stone RM, Maguire M, Rosenthal DS et al. Is heparin administration necessary during induction chemotherapy for patients with acute promyelocytic leukemia? Blood 1987; 69: 187-191.

24 Collins AJ, Bloomfield CD, Peterson BA, McKenna RW, Edson JR. Acute promyelocytic leukemia. Management of the coagulopathy during daunorubicinprednisone remission induction. Arch Intern Med 1978; 138: 1677-1680.

25 Leone G, Mango G, Alfano G. Disseminated intravascular coagulation in acute promyelocytic leukemia. Possibility of treatment with glucocorticoids at high doses. Nouv Rev Fr Hematol 1978; 20: 395-401.

26 Daly PA, Schiffer CA, Wiernik PH. Acute promyelocytic leukemia-clinical management of 15 patients. Am J Hematol 1980; 8: 347-359.

27 Avvisati G, Petti MC, Petrucci MT, Falconi E, Tirindelli MC, Mandelli F. Treatment of recurrent promyelocytic leukemia with a combination regimen utilizing amsacrine, cytosine arabinoside and 6-thioguanine (AAT). Haematologica 1989; 74: 279-282.

28 Marty M, Ganem G, Fischer J, Flandrin G, Berger R, Schaison G et al. [Acute promyelocytic leukemia: retrospective study of 119 patients treated with daunorubicin]. Nouv Rev Fr Hematol 1984; 26: 371-378.

29 Wang ZY, Chen Z. Acute promyelocytic leukemia: from highly fatal to highly curable. Blood 2008; 111: 2505-2515.

30 Tallman MS, Andersen JW, Schiffer CA, Appelbaum FR, Feusner JH, Ogden A et al. All-trans-retinoic acid in acute promyelocytic leukemia. N Engl J Med 1997; 337: 1021-1028.

31 Chen ZX, Xue YQ, Zhang R, Tao RF, Xia XM, Li C et al. A clinical and experimental study on all-trans retinoic acid-treated acute promyelocytic leukemia patients. Blood 1991; 78: 1413-1419.

32 Castaigne S, Chomienne C, Daniel MT, Ballerini P, Berger R, Fenaux P et al. All-trans retinoic acid as a differentiation therapy for acute promyelocytic leukemia. I. Clinical results. Blood 1990; 76: 1704-1709.

33 Huang ME, Ye YC, Chen SR, Chai JR, Lu JX, Zhoa L et al. Use of all-trans retinoic acid in the treatment of acute promyelocytic leukemia. Blood 1988; 72: 567-572.

34 Fenaux P, Chastang C, Chevret S, Sanz M, Dombret H, Archimbaud E et al. A randomized comparison of all transretinoic acid (ATRA) followed by chemotherapy and ATRA plus chemotherapy and the role of maintenance therapy in newly diagnosed acute promyelocytic leukemia. The European APL Group. Blood 1999; 94: 1192-1200.

35 Lengfelder E, Reichert A, Schoch C, Haase D, Haferlach T, Loffler H et al. Double induction strategy including high dose cytarabine in combination with all-trans retinoic acid: effects in patients with newly diagnosed acute promyelocytic leukemia. German AML Cooperative Group. Leukemia 2000; 14: 1362-1370.

36 Mandelli F, Diverio D, Avvisati G, Luciano A, Barbui T, Bernasconi C et al. Molecular remission in PML/RAR alpha-positive acute promyelocytic leukemia by combined all-trans retinoic acid and idarubicin (AIDA) therapy. Gruppo ItalianoMalattie Ematologiche Maligne dell'Adulto and Associazione Italiana di Ematologia ed Oncologia Pediatrica Cooperative Groups. Blood 1997; 90: 1014-1021.
37 Asou N, Adachi K, Tamura J, Kanamaru A, Kageyama S, Hiraoka A et al. Analysis of prognostic factors in newly diagnosed acute promyelocytic leukemia treated with all-trans retinoic acid and chemotherapy. Japan Adult Leukemia Study Group. J Clin Oncol 1998; 16: 78-85.

38 Sanz MA, Martin G, Rayon C, Esteve J, Gonzalez M, Diaz-Mediavilla J et al. A modified AIDA protocol with anthracycline-based consolidation results in high antileukemic efficacy and reduced toxicity in newly diagnosed PML/ RARalpha-positive acute promyelocytic leukemia. PETHEMA group. Blood 1999; 94: 3015-3021.

39 Fenaux P, Le Deley MC, Castaigne S, Archimbaud E, Chomienne C, Link H et al. Effect of all transretinoic acid in newly diagnosed acute promyelocytic leukemia. Results of a multicenter randomized trial. European APL 91 Group. Blood 1993; 82: 3241-3249.

40 de Botton S, Chevret S, Coiteux V, Dombret H, Sanz M, San Miguel J et al. Early onset of chemotherapy can reduce the incidence of ATRA syndrome in newly diagnosed acute promyelocytic leukemia (APL) with low white blood cell counts: results from APL 93 trial. Leukemia 2003; 17: 339-342.

41 Ades L, Chevret S, Raffoux E, de Botton S, Guerci A, Pigneux A et al. Is cytarabine useful in the treatment of acute promyelocytic leukemia? Results of a randomized trial from the European Acute Promyelocytic Leukemia Group. J Clin Oncol 2006; 24: 5703-5710.

42 Burnett AK, Hills RK, Grimwade D, Goldstone AH, Hunter A, Milligan D et al. Idarubicin and ATRA is as effective as MRC chemotherapy in patients with acute promyelocytic leukaemia with lower toxicity and resource usage: preliminary results of the MRC AML15 Trial. ASH Annual Meeting Abstracts. 2007; 110: 589.

43 Ades L, Raffoux E, Chevret S, de Botton S, Guerci A, Pigneux A et al. Is $\mathrm{AraC}$ required in the treatment of standard risk $\mathrm{APL}$ ? Long term results of a randomized trial (APL 2000) from the French Belgian Swiss APL Group. ASH Annual Meeting Abstracts 2010; 116: 13.

44 Avvisati G, Lo Coco F, Diverio D, Falda M, Ferrara F, Lazzarino M et al. AIDA (all-trans retinoic acid+idarubicin) in newly diagnosed acute promyelocytic leukemia: a Gruppo Italiano Malattie Ematologiche Maligne dell'Adulto (GIMEMA) pilot study. Blood 1996; 88: 1390-1398.

45 Sanz MA, Martin G, Gonzalez M, Leon A, Rayon C, Rivas C et al. Risk-adapted treatment of acute promyelocytic leukemia with all-trans-retinoic acid and anthracycline monochemotherapy: a multicenter study by the PETHEMA group. Blood 2004; 103: 1237-1243.

46 Sanz MA, Montesinos P, Rayon C, Holowiecka A, de la Serna J, Milone G et al. Risk-adapted treatment of acute promyelocytic leukemia based on all-trans retinoic acid and anthracycline with addition of cytarabine in consolidation therapy for high-risk patients: further improvements in treatment outcome. Blood 2010; 115: 5137-5146.

47 Iland HJ, Bradstock K, Supple SG, Catalano A, Collins M, Hertzberg M et al. All-trans-retinoic acid, idarubicin, and IV arsenic trioxide as initial therapy in acute promyelocytic leukemia (APML4). Blood 2012; 120: 1570-1580, quiz 752.

48 Niu C, Yan H, Yu T, Sun HP, Liu JX, Li XS et al. Studies on treatment of acute promyelocytic leukemia with arsenic trioxide: remission induction, follow-up, and molecular monitoring in 11 newly diagnosed and 47 relapsed acute promyelocytic leukemia patients. Blood 1999; 94: 3315-3324.

49 Zheng PZ, Wang KK, Zhang QY, Huang QH, Du YZ, Zhang QH et al. Systems analysis of transcriptome and proteome in retinoic acid/arsenic trioxide-induced cell differentiation/apoptosis of promyelocytic leukemia. Proc Natl Acad Sci USA 2005; 102: 7653-7658.

50 Gianni M, Koken MH, Chelbi-Alix MK, Benoit G, Lanotte M, Chen Z et al. Combined arsenic and retinoic acid treatment enhances differentiation and apoptosis in arsenic-resistant NB4 cells. Blood 1998; 91: 4300-4310.

51 Dos Santos GA, Kats L, Pandolfi PP. Synergy against PML-RARa: targeting transcription, proteolysis, differentiation, and self-renewal in acute promyelocytic leukemia. J Exp Med 2013; 210: 2793-2802.

52 Nasr R, Guillemin MC, Ferhi O, Soilihi H, Peres L, Berthier C et al. Eradication of acute promyelocytic leukemia-initiating cells through PML-RARA degradation. Nat Med 2008; 14: 1333-1342.

53 Shen ZX, Shi ZZ, Fang J, Gu BW, Li JM, Zhu YM et al. All-trans retinoic acid/As2O3 combination yields a high quality remission and survival in newly diagnosed acute promyelocytic leukemia. Proc Natl Acad Sci USA 2004; 101: 5328-5335.

54 Iland HJ, Collins M, Hertzberg MS, Seldon M, Grigg AP, Firkin F et al. Final analysis of the Australasian Leukaemia and Lymphoma Group (ALLG) APML4 Trial: all-trans retinoic acid (ATRA), intravenous arsenic trioxide (ATO) and idarubicin (IDA) as initial therapy for acute promyelocytic leukemia (APL). American Society of Hematology Meeting, San Francisco, CA, USA, 2014.

55 Estey E, Garcia-Manero G, Ferrajoli A, Faderl S, Verstovsek S, Jones D et al. Use of all-trans retinoic acid plus arsenic trioxide as an alternative to chemotherapy in untreated acute promyelocytic leukemia. Blood 2006; 107: 3469-3473.

56 Ravandi F, Estey EH, Cortes JE, O'Brien S, Pierce SA, Brandt M et al. Phase II study of all-trans retinoic acid (ATRA), arsenic trioxide (ATO), with or without 
gemtuzumab ozogamicin (GO) for the frontline therapy of patients with acute promyelocytic leukemia (APL)Blood (ASH Annual Meeting Abstracts) 2010; 116: Abstract 1080

57 Pellicori P, Calicchia A, Lococo F, Cimino G, Torromeo C. Subclinical anthracycline cardiotoxicity in patients with acute promyelocytic leukemia in long-term remission after the AIDA protocol. Congest Heart Fail (Greenwich, Conn) 2012; 18: 217-221.

58 Andersen MK, Pedersen-Bjergaard J. Therapy-related MDS and AML in acute promyelocytic leukemia. Blood 2002; 100: 1928-1929, author reply 9.

59 Garcia-Manero G, Kantarjian HM, Kornblau S, Estey E. Therapy-related myelodysplastic syndrome or acute myelogenous leukemia in patients with acute promyelocytic leukemia (APL). Leukemia 2002; 16: 1888

60 Latagliata R, Petti MC, Fenu S, Mancini M, Spiriti MA, Breccia M et al. Therapy-related myelodysplastic syndrome-acute myelogenous leukemia in patients treated for acute promyelocytic leukemia: an emerging problem. Blood 2002; 99: 822-824.

61 Montesinos P, Gonzalez JD, Gonzalez J, Rayon C, de Lisa E, Amigo ML et al. Therapy-related myeloid neoplasms in patients with acute promyelocytic leukemia treated with all-trans-retinoic Acid and anthracycline-based chemotherapy. J Clin Oncol 2010; 28: 3872-3879.

62 Platzbecker U, Avvisati G, Ehninger G, Cicconi L, Thiede C, Ferrara F et al. Improved outcome with ATRA-arsenic trioxide compared to ATRAchemotherapy in non-high risk acute promyelocytic leukemia - updated results of the Italian-German APL0406 Trial on the Extended Final Series. American Society of Hematology Meeting, San Francisco, CA, USA, 2014

63 Lo-Coco F, Avvisati G, Vignetti M, Thiede C, Orlando SM, lacobelli S et al. Retinoic acid and arsenic trioxide for acute promyelocytic leukemia. N Engl J Med 2013; 369: 111-121.

64 Efficace F, Mandelli F, Avvisati G, Cottone F, Ferrara F, Di Bona E et al. Randomized phase III trial of retinoic acid and arsenic trioxide versus retinoic acid and chemotherapy in patients with acute promyelocytic leukemia: healthrelated quality-of-life outcomes. J Clin Oncol 2014; 32: 3406-3412.

65 Eghtedar A, Rodriguez I, Kantarjian H, O'Brien S, Daver N, Garcia-Manero G et al. Incidence of secondary neoplasms in patients with acute promyelocytic leukemia treated with all-trans retinoic acid plus chemotherapy or with all-trans retinoic acid plus arsenic trioxide. Leuk Lymph 2014; e-pub ahead of print 3 November 2014; doi:10.3109/10428194.2014.953143.

66 Lo-Coco F, Avvisati G, Vignetti M, Breccia M, Gallo E, Rambaldi A et al. Front-line treatment of acute promyelocytic leukemia with AIDA induction followed by risk-adapted consolidation for adults younger than 61 years: results of the AIDA-2000 trial of the GIMEMA Group. Blood 2010; 116: 3171-3179.

67 Flanagan SA, Meckling KA. All- trans-retinoic acid increases cytotoxicity of 1-beta-D-arabinofuranosylcytosine in NB4 cells. Cancer Chemother Pharmacol 2003; 51: 363-375.

68 Powell BL, Moser B, Stock W, Gallagher RE, Willman CL, Stone RM et al. Arsenic trioxide improves event-free and overall survival for adults with acute promyelocytic leukemia: North American Leukemia Intergroup Study C9710. Blood 2010; 116: 3751-3757.

69 Gore SD, Gojo I, Sekeres MA, Morris L, Devetten M, Jamieson K et al. Single cycle of arsenic trioxide-based consolidation chemotherapy spares anthracycline exposure in the primary management of acute promyelocytic leukemia. J Clin Oncol 2010; 28: 1047-1053.

70 Ravandi F, Estey E, Jones D, Faderl S, O'Brien S, Fiorentino J et al. Effective treatment of acute promyelocytic leukemia with all-trans-retinoic acid, arsenic trioxide, and gemtuzumab ozogamicin. J Clin Oncol 2009; 27: 504-510.

71 Muchtar E, Vidal L, Ram R, Gafter-Gvili A, Shpilberg O, Raanani P. The role of maintenance therapy in acute promyelocytic leukemia in the first complete remission. Cochrane Database Syst Rev 2013; 3: Cd009594.

72 Coutre SE, Othus M, Powell B, Willman CL, Stock W, Paietta E et al. Arsenic trioxide during consolidation for patients with previously untreated low/ intermediate risk acute promyelocytic leukaemia may eliminate the need for maintenance therapy. Br J Haematol 2014; 165: 497-503.

73 Breccia M, Minotti C, Latagliata R, Loglisci G, Salaroli A, Loglisci MG et al. Influence of time to complete remission and duration of all-trans retinoic acid therapy on the relapse risk in patients with acute promyelocytic leukemia receiving AIDA protocols. Leuk Res 2013; 37: 383-385.

74 Licht JD, Chomienne C, Goy A, Chen A, Scott AA, Head DR et al. Clinical and molecular characterization of a rare syndrome of acute promyelocytic leukemia associated with translocation $(11 ; 17)$. Blood 1995; 85: 1083-1094.

$75 \mathrm{Zhu} \mathrm{HH}$, Qin YZ, Huang XJ. Resistance to arsenic therapy in acute promyelocytic leukemia. N Engl J Med 2014; 370: 1864-1866.

76 Tallman MS. Treatment of relapsed or refractory acute promyelocytic leukemia. Best Pract Res Clin Haematol 2007; 20: 57-65.

77 Specchia G, Lo Coco F, Vignetti M, Avvisati G, Fazi P, Albano F et al. Extramedullary involvement at relapse in acute promyelocytic leukemia patients treated or not with all-trans retinoic acid: a report by the Gruppo Italiano Malattie Ematologiche dell'Adulto. J Clin Oncol 2001; 19: 4023-4028.

78 Lou Y, Suo S, Tong Y, Tong H, Qian W, Meng H et al. Outcomes and prognostic factors of first relapsed acute promyelocytic leukemia patients undergoing salvage therapy with intravenous arsenic trioxide and chemotherapy. Ann Hematol 2014; 93: 941-948.

79 Pemmaraju N, Tanaka MF, Ravandi F, Lin H, Baladandayuthapani V, Rondon $\mathrm{G}$ et al. Outcomes in patients with relapsed or refractory acute promyelocytic leukemia treated with or without autologous or allogeneic hematopoietic stem cell transplantation. Clin Lymphoma Myeloma Leuk 2013; 13: 485-492.

80 Yanada M, Tsuzuki M, Fujita H, Fujimaki K, Fujisawa S, Sunami K et al. Phase 2 study of arsenic trioxide followed by autologous hematopoietic cell transplantation for relapsed acute promyelocytic leukemia. Blood 2013; 121 3095-3102.

81 Holter Chakrabarty JL, Rubinger M, Le-Rademacher J, Wang HL, Grigg A Selby GB et al. Autologous is superior to allogeneic hematopoietic cell transplantation for acute promyelocytic leukemia in second complete remission. Biol Blood Marrow Transplant 2014; 20: 1021-1025.

82 Mannis GN, Logan AC, Leavitt AD, Yanada M, Hwang J, Olin RL et al. Delayed hematopoietic recovery after auto-SCT in patients receiving arsenic trioxide-based therapy for acute promyelocytic leukemia: a multi-center analysis. Bone Marrow Transplant 2014; 50: 40-44.

83 Micol JB, Raffoux E, Boissel N, Lengline E, Canet E, Daniel MT et al. Management and treatment results in patients with acute promyelocytic leukaemia (APL) not enrolled in clinical trials. Eur J Cancer2014; 50: 1159-1168.

84 Lengfelder E, Hofmann WK, Nolte F. Management of elderly patients with acute promyelocytic leukemia: progress and problems. Ann Hematol 2013; 92 1181-1188.

85 Park JH, Qiao B, Panageas KS, Schymura MJ, Jurcic JG, Rosenblat TL et al. Early death rate in acute promyelocytic leukemia remains high despite all-trans retinoic acid. Blood 2011; 118: 1248-1254.

86 Iland HJ, Seymour JF, Wei A. Optimal approach for high-risk acute promyelocytic leukemia. Curr Opin Hematol 2014; 21: 102-113.

87 Altman JK, Rademaker A, Cull E, Weitner BB, Ofran Y, Rosenblat TL et al. Administration of ATRA to newly diagnosed patients with acute promyelocytic leukemia is delayed contributing to early hemorrhagic death. Leuk Res 2013; 37: 1004-1009.

88 Rashidi A, Riley M, Goldin TA, Sayedian F, Bayerl MG, Aguilera NS et al. Delay in the administration of all-trans retinoic acid and its effects on early mortality in acute promyelocytic leukemia: final results of a multicentric study in the United States. Leuk Res 2014; 38: 1036-1040.

89 Tallman MS, Altman JK. How I treat acute promyelocytic leukemia. Blood 2009; 114: $5126-5135$.

90 Grisariu S, Spectre G, Kalish Y, Gatt ME. Increased risk of central venous catheterassociated thrombosis in acute promyelocytic leukemia: a single-institution experience. Eur J Haematol 2013; 90: 397-403.

91 Kumana CR, Au WY, Lee NS, Kou M, Mak RW, Lam CW et al. Systemic availability of arsenic from oral arsenic-trioxide used to treat patients with hematological malignancies. Eur J Clin Pharmacol 2002; 58: 521-526.

92 Au WY, Kumana CR, Kou M, Mak R, Chan GC, Lam CW et al. Oral arsenic trioxide in the treatment of relapsed acute promyelocytic leukemia. Blood 2003; 102 407-408.

93 Siu CW, Au WY, Yung C, Kumana CR, Lau CP, Kwong YL et al. Effects of oral arsenic trioxide therapy on QT intervals in patients with acute promyelocytic leukemia: implications for long-term cardiac safety. Blood 2006; 108: 103-106.

94 Au WY, Kumana CR, Lee HK, Lin SY, Liu H, Yeung DY et al. Oral arsenic trioxidebased maintenance regimens for first complete remission of acute promyelocytic leukemia: a 10-year follow-up study. Blood 2011; 118: 6535-6543.

$95 \mathrm{Zhu} \mathrm{HH}$, Wu DP, Jin J, Li JY, Ma J, Wang JX et al. Oral tetra-arsenic tetra-sulfide formula versus intravenous arsenic trioxide as first-line treatment of acute promyelocytic leukemia: a multicenter randomized controlled trial. J Clin Oncol 2013; 31: 4215-4221.

96 Zeidan AM, Gore SD. New strategies in acute promyelocytic leukemia: moving to an entirely oral, chemotherapy-free upfront management approach. Clin Cance Res 2014; 20: 4985-4993.

97 Takeshita A, Shinjo K, Naito K, Matsui H, Sahara N, Shigeno K et al. Efficacy of gemtuzumab ozogamicin on ATRA- and arsenic-resistant acute promyelocytic leukemia (APL) cells. Leukemia 2005; 19: 1306-1311.

98 Castaigne S, Pautas C, Terre C, Raffoux E, Bordessoule D, Bastie JN et al. Effect of gemtuzumab ozogamicin on survival of adult patients with de-novo acute myeloid leukaemia (ALFA-0701): a randomised, open-label, phase 3 study. Lancet 2012; 379: 1508-1516.

99 Ravandi F, Estey EH, Appelbaum FR, Lo-Coco F, Schiffer CA, Larson RA et al. Gemtuzumab ozogamicin: time to resurrect? J Clin Oncol 2012; 30: 3921-3923. 
100 Stein EM, Stein A, Walter RB, Fathi AT, Lancet JE, Kovacsovics TJ et al. Interim analysis of a phase 1 trial of SGN-CD33A in patients with CD33-positive acute myeloid leukemia (AML). American Society of Hematology Meeting, San Francisco, CA, USA, 2014.

101 Lo-Coco F, Cimino G, Breccia M, Noguera NI, Diverio D, Finolezzi E et al. Gemtuzumab ozogamicin (Mylotarg) as a single agent for molecularly relapsed acute promyelocytic leukemia. Blood 2004; 104: 1995-1999.

102 Hashimoto Y, Kagechika H, Kawachi E, Fukasawa H, Saito G, Shudo K. Correlation of differentiation-inducing activity of retinoids on human leukemia cell lines HL-60 and NB4. J Cancer Res Clin Oncol 1995; 121: 696-698.

103 Tobita T, Takeshita A, Kitamura K, Ohnishi K, Yanagi M, Hiraoka A et al. Treatment with a new synthetic retinoid, Am80, of acute promyelocytic leukemia relapsed from complete remission induced by all-trans retinoic acid. Blood 1997; 90: 967-973.

104 Shinagawa K, Yanada M, Sakura T, Ueda Y, Sawa M, Miyatake J et al. Tamibarotene as maintenance therapy for acute promyelocytic leukemia: results from a randomized controlled trial. J Clin Oncol 2014; 32: 3729-3735.
105 Sanford D, LoCoco F, Sanz MA, Di Bona E, Coutre S, Altman JK et al. Single agent tamibarotene has activity in acute promyelocytic leukemia patients previously treated with ATRA and arsenic trioxide, but does not produce durable responses. American Society of Hematology Meeting, San Francisco, CA, USA, 2014.

106 Shetty AV, Ravandi F, Alapati N, Borthakur G, Garcia-Manero G, Kadia TM et al. Survivorship in APL- outcomes of acute promyelocytic leukemia (APL) patients (pts) after maintaining complete remission (CR) for at least 3 years. American Society of Hematology Meeting, San Francisco, CA, USA, 2014.

\section{(c) (i)}

This work is licensed under a Creative Commons Attribution 4.0 International License. The images or other third party material in this article are included in the article's Creative Commons license, unless indicated otherwise in the credit line; if the material is not included under the Creative Commons license, users will need to obtain permission from the license holder to reproduce the material. To view a copy of this license, visit http://creativecommons. org/licenses/by/4.0/ 